\title{
Bridging molecular dynamics and correlated wave-function methods for accurate finite-temperature properties
}

\author{
Dario Rocca, ${ }^{1,2, *}$ Anant Dixit, ${ }^{1,2}$ Michael Badawi, ${ }^{1,2}$ Sébastien Lebègue, ${ }^{1,2}$ Tim Gould, ${ }^{3}$ and Tomáš Bučko ${ }^{4,5, \dagger}$ \\ ${ }^{1}$ Université de Lorraine, LPCT, UMR 7019, F-54506 Vandouvre-lès-Nancy, France \\ ${ }^{2}$ CNRS, LPCT, UMR 7019, F-54506 Vandouvre-lès-Nancy, France \\ ${ }^{3}$ Qld Micro- and Nanotechnology Centre, Griffith University, Nathan, Queensland 4111, Australia \\ ${ }^{4}$ Department of Physical and Theoretical Chemistry, Faculty of Natural Sciences, Comenius University in Bratislava, \\ Ilkovičova 6, SK-84215 Bratislava, Slovakia \\ ${ }^{5}$ Institute of Inorganic Chemistry, Slovak Academy of Sciences, Dúbravská cesta 9, SK-84236 Bratislava, Slovakia
}

(Received 22 September 2018; revised manuscript received 10 February 2019; published 25 April 2019)

\begin{abstract}
We introduce the "selPT" perturbative approach, based on ab initio molecular dynamics (AIMD), for computing accurate finite-temperature properties by efficiently using correlated wave-function methods. We demonstrate the power of the method by computing prototypical molecular enthalpies of adsorption in zeolite $\left(\mathrm{CH}_{4}\right.$ and $\mathrm{CO}_{2}$ on protonated chabazite at $\left.300 \mathrm{~K}\right)$ using the random phase approximation. Results are in excellent agreement with experiment. The improved accuracy provided by selPT represents a crucial step towards the goal of truly quantitative AIMD predictions of experimental observables at finite temperature.
\end{abstract}

DOI: 10.1103/PhysRevMaterials.3.040801

Since the seminal work of Car and Parrinello [1], the field of $a b$ initio molecular dynamics (AIMD) has become impressively popular as it offers insights into finite-temperature systems. Because of the high computational cost involved in $a b$ initio electronic structure calculations, AIMD is usually based on density functional theory (DFT) [2], which provides a good compromise between accuracy and computational cost. However, approximate DFT functionals do not systematically reach chemical accuracy $(1 \mathrm{kcal} / \mathrm{mol})$ and are affected by a series of known shortcomings [3]. This can hamper the understanding of systems for which DFT approaches are insufficient to obtain a quantitative, or even qualitative, standard.

An alternative to DFT is to use correlated wave-function methods, which are more accurate and systematically improvable, but involve a higher computational cost. For applications to molecular systems, the Møller-Plesset perturbation theory [4] (MP2, MP3, etc.) and coupled-cluster theory [5] (CC) have long been used [6]. For condensed phase applications, MP2 has only recently appeared-using plane-wave [7,8], localized [9], and hybrid [10] basis sets. Approximations based on coupled-cluster theory have been implemented in the VASP [11] and PYSCF codes [12,13].

A particularly promising correlated approach for condensed phase applications is the random phase approximation (RPA) [14-21]. This approach to the ground state electronic correlation energy can be seen both as a DFT approximation [16,22] or a CC approximation [23]. The RPA also has analogies with MP theory [24]. Unlike CC and MP theories, which are applied exclusively as post Hartree-Fock methods, the RPA is most often evaluated from a DFT starting point.

\footnotetext{
*dario.rocca@univ-lorraine.fr

†bucko19@uniba.sk
}

The success of the RPA is largely related to its accurate description of weak van der Waals (vdW) interactions [17,18], but this approximation is sometimes less reliable for shortrange interactions. Although significantly more expensive than traditional DFT approximations, the RPA can be performed for systems with up to a few hundred electrons and a series of applications to condensed phase systems have been reported in the literature [25-34]. When extra precision is required, beyond-RPA methods can be used [24,35-43].

Whether via RPA, MP, or CC theories, correlated wavefunction methods hold the promise of a significantly higher level of accuracy, which is of fundamental importance for a design of truly predictive approaches to assist experimental and technological developments. However, they are characterized by an unfavorable scaling, slow basis set convergence $[26,28,44]$, and slow cell size convergence [45], making them expensive.

Despite these difficulties, high-level methodologies are of vital importance in many technologically attractive cases involving, e.g., nanoporous materials such as zeolites [46-50] or metal-organic frameworks [49-52]. These materials are intensively investigated for their ability to selectively adsorb target compounds and this property is used in numerous applications such as depollution [46-51] or separation of chemicals [52,53]. Reliable predictions of adsorption properties of these materials can be made only if the electronic structure method used in simulations accurately describes all interactions within the system of interest, and if the thermal effects are properly accounted for by using a suitable statistical mechanics method such as molecular dynamics (MD) or Monte Carlo. Unfortunately, simulations combining highlevel methodologies with MD or Monte Carlo approaches, usually requiring ensembles of tens of thousands of configurations, are thus far beyond the reach of the computational power available today. In fact, the forces for correlated 
methods in the condensed phase have only recently been implemented [54,55] and employed in MD simulations [25,56].

In this Rapid Communication we present a methodology based on perturbation theory that extends the applicability of correlated methods to compute finite-temperature properties at a reasonable computational cost. Our approach starts from MD simulations based on computationally inexpensive DFT functionals. Then, a small number (a few tens) of significant configurations are chosen from the ensemble of structures generated by MD to represent the full probability distribution. Finally, correlated wave-function calculations are performed on these configurations and the results are used to reconstruct the corresponding probability distribution and ensemble averages. Below, we will apply this method to the computation of the finite-temperature adsorption enthalpies of $\mathrm{CH}_{4}$ and $\mathrm{CO}_{2}$ in the zeolite chabazite, using RPA.

The approach introduced in this Rapid Communication will be referred to as perturbation theory on selected configurations (selPT). A detailed discussion of perturbation theory in the context of molecular dynamics and free-energy calculations can be found in Ref. [57]. The main ideas and working equations will be summarized here.

Within the canonical ensemble, the expectation value of a certain observable $O$ is defined as follows: $\langle O\rangle_{H}=\int O(\mathbf{q}, \mathbf{p}) \exp \{-\beta H(\mathbf{q}, \mathbf{p})\} d \mathbf{q} d \mathbf{p} / Z, \quad$ for $\quad Z=$ $\int \exp \{-\beta H(\mathbf{q}, \mathbf{p})\} d \mathbf{q} d \mathbf{p}$. Here, $\beta=1 / k_{B} T$ and $\mathbf{q}$ and p denote nuclear positions and momenta, respectively. In this work, the notation $\langle\cdots\rangle_{H}$ represents the canonical ensemble average corresponding to the classical Hamiltonian $H(\mathbf{q}, \mathbf{p})=T(\mathbf{p})+V(\mathbf{q})$, with $T(\mathbf{p})$ and $V(\mathbf{q})$ being the nuclear kinetic and potential energies, respectively.

Exploring all $\mathbf{p}$ and $\mathbf{q}$ is generally impossible. An alternative is to assume the ergodic hypothesis. Then, the ensemble average can more usefully be expressed as

$$
\langle O\rangle_{H}=\lim _{\tau \rightarrow \infty} \frac{1}{\tau} \int_{0}^{\tau} O[\mathbf{q}(t), \mathbf{p}(t)] d t,
$$

a temporal average over an AIMD trajectory $\dot{\mathbf{q}}=\nabla_{\mathbf{p}} H, \dot{\mathbf{p}}=$ $-\nabla_{\mathbf{q}} H$. For infinite time, $\langle O\rangle_{H}$ is independent of trajectory, since the system explores all configurations. However, for finite time, the choice of trajectory is crucial.

Ideally, we could use any method to obtain long AIMD trajectories that ensure a sufficient level of statistical precision. AIMD simulations are time consuming, however, and thus long trajectories require a numerically efficient model electronic energy Hamiltonian $\hat{H}_{e}$, with potential

$$
V(\mathbf{q})=\left\langle\Psi(\mathbf{q})\left|\hat{H}_{e}\right| \Psi(\mathbf{q})\right\rangle+U_{I I}(\mathbf{q}),
$$

at nuclear configuration $\mathbf{q}$ and repulsive nuclear energy $U_{I I}$. Unfortunately, this leaves us with a compromise between accuracy (quality of electronic energies) and efficiency (length of trajectories), for a given precision.

In such cases, perturbation theory can be used to transform from an efficient/inaccurate Hamiltonian $H=T+V$ (e.g. PBE+D2, a dispersion corrected version of the Perdew Burke Ernzerhof functional [58]) to an expensive/accurate Hamiltonian $H^{\prime}$ (e.g., RPA), via the perturbation

$$
H^{\prime}(\mathbf{q}, \mathbf{p})=H-V+V^{\prime} \equiv H(\mathbf{q}, \mathbf{p})+\Delta V(\mathbf{q}) .
$$
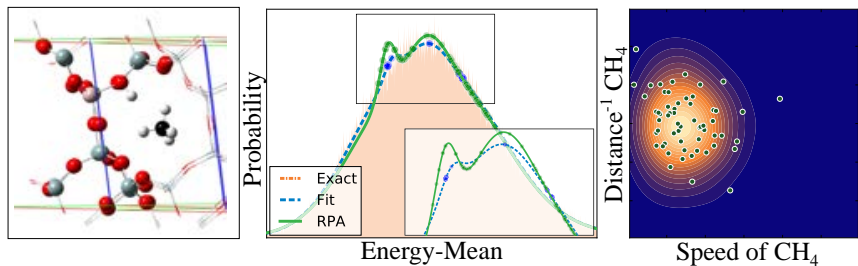

FIG. 1. Left: The model of $\mathrm{CH}_{4}$ adsorbed on protonated chabazite studied in this work. Color code: $\mathrm{Al}$ in pink, $\mathrm{O}$ in red, $\mathrm{Si}$ in blue, $\mathrm{C}$ in black, and $\mathrm{H}$ in white. Middle: Probability distributions of the energy for $\mathrm{CH}_{4}$ in protonated chabazite at $T=300 \mathrm{~K}$. Compares the exact distribution (2000 bins, shaded orange) from a PBE+D2 MD run, with its selPT counterpart obtained using 60 configurations (SET2@PBE+D2, blue dashed line), and perturbed onto RPA (green solid line). All distributions are shown relative to their mean. Right: Phase space of the PBE+D2 trajectory projected on $\mathrm{CH}_{4}$ speed and a metric representing the inverse distance of the $\mathrm{CH}_{4}$ from the chabazite. The 60 selPT states are shown as circles in both panels.

The trick is to write thermal averages of potentials as

$$
\langle V\rangle_{H}=\int E d E P_{H}^{V}(E), \quad P_{H}^{V}(E) \equiv\langle\delta(V-E)\rangle_{H},
$$

where $P_{H}^{V}(E)$ describes the energy $E$ distribution of some potential energy $V$ of interest. The subscript $H$ indicates that it is calculated in the trajectory described by Hamiltonian $H$. Then, one uses the perturbation $\Delta V(\mathbf{q})$ to obtain $\left\langle V^{\prime}\right\rangle_{H^{\prime}}=$ $\int E d E P_{H^{\prime}}^{V^{\prime}}(E)$ from

$$
P_{H^{\prime}}^{V^{\prime}}(E)=\frac{\left\langle\delta\left(V^{\prime}-E\right) \exp \{-\beta \Delta V\}\right\rangle_{H}}{\langle\exp \{-\beta \Delta V\}\rangle_{H}} .
$$

$P_{H^{\prime}}^{V^{\prime}}$ then gives properties of the trajectory $H^{\prime}$, but is calculated on the trajectory $H$.

This lets us define properties of the system driven by the primed Hamiltonian (e.g., RPA) in terms of trajectories [Eq. (1)] on the system described by the original Hamiltonian (e.g., PBE+D2). Typical calculations employing perturbative approaches based on this formula involve large (albeit reduced) numbers of configurations computed in a given MD or Monte Carlo simulation, which are implausible for the expensive methods we seek to use. In this work, we thus introduce an innovation that lets the perturbation be evaluated with very few additional calculations, but without significant loss of precision. We thereby break the compromise between speed and accuracy that hampers the application of AIMD to difficult problems, such as the ones presented below.

To understand the challenges involved in the direct application of perturbation theory, we now present a specific numerical example that will also be used later to discuss our methodological developments. Let us consider the finitetemperature adsorption of methane on protonated chabazite (later, results will also be presented for the adsorption of carbon dioxide). The model used for this system, which involves 200 valence electrons per unit cell, is shown in Fig. 1 and further discussed in the Supplemental Material [59]. Using MD in the $N V T$ ensemble, the adsorption enthalpy can be computed as

$$
\Delta_{\mathrm{ads}} H(T)=\left\langle V_{S+A}\right\rangle_{H}-\left[\left\langle V_{S}\right\rangle_{H}+\left\langle V_{A}\right\rangle_{H}\right]-k_{B} T,
$$


TABLE I. Enthalpy of adsorption $\Delta_{\text {ads }} H(T)$ (in $\mathrm{kcal} / \mathrm{mol}$ ) of $\mathrm{CH}_{4}$ in protonated chabazite at $300 \mathrm{~K}$ evaluated at the PBE+D2, PBE, and RPA levels of theory. Results are calculated using full MD trajectories (MD@PBE and MD@PBE+D2) applying the selPT method to a set with $N_{G}=60$ configurations (SET1@PBE+D2), $N_{G}=120(\mathrm{SET} 2+3 @ \mathrm{PBE}+\mathrm{D} 2)$, and $N_{G}=180(\mathrm{SET} 1+2+3 @ \mathrm{PBE}+\mathrm{D} 2)$. Experimental results at room temperature give $\Delta_{\text {ads }} H(T)=-4.06 \mathrm{kcal} / \mathrm{mol}[62,63]$.

\begin{tabular}{|c|c|c|c|c|c|}
\hline \multirow[b]{2}{*}{$V$} & \multicolumn{5}{|c|}{$H$} \\
\hline & $\begin{array}{c}\mathrm{MD} \\
@ \mathrm{PBE}\end{array}$ & $\begin{array}{c}\mathrm{MD} \\
@ \mathrm{PBE}+\mathrm{D} 2\end{array}$ & $\begin{array}{c}\text { SET1 } \\
@ \text { PBE+D2 }\end{array}$ & $\begin{array}{c}\mathrm{SET} 2+3 \\
@ \mathrm{PBE}+\mathrm{D} 2\end{array}$ & $\begin{array}{l}\mathrm{SET} 1+2+3 \\
@ \mathrm{PBE}+\mathrm{D} 2\end{array}$ \\
\hline $\mathrm{PBE}+\mathrm{D} 2$ & $-5.72 \pm 0.48$ & $-6.09 \pm 0.18$ & & & \\
\hline PBE & $-1.13 \pm 0.18$ & $-1.11 \pm 0.25$ & $-1.31 \pm 0.58$ & $-1.84 \pm 0.53$ & $-1.73 \pm 0.43$ \\
\hline RPA & & & $-4.20 \pm 0.62$ & $-4.45 \pm 0.48$ & $-4.38 \pm 0.39$ \\
\hline
\end{tabular}

where subscripts $S$ and $A$ denote the clean substrate (protonated chabazite), and the adsorbate (methane), respectively, while $S+A$ indicates the system of substrate interacting with adsorbate. In order to evaluate the ensemble averages in Eq. (6), MD simulations at $300 \mathrm{~K}$ have been performed with the VASP code [60] using two different approximations, namely, $\mathrm{PBE}$ and $\mathrm{PBE}+\mathrm{D} 2$ (a vdW corrected version of the PBE functional [58]). Computational details are provided in Supplemental Material [59]. These two MD simulations will be denoted as MD@PBE and MD@PBE+D2, respectively.

In Table I, the PBE (PBE+D2) enthalpy of adsorption corresponding to MD@PBE (MD@PBE+D2) is an "exact" estimate, namely, an estimate where the averaged potential and the Hamiltonian used for the sampling are at the same level of theory; the PBE $(\mathrm{PBE}+\mathrm{D} 2)$ value evaluated from $\mathrm{MD} @ \mathrm{PBE}+\mathrm{D} 2$ (MD@PBE) is based on the perturbative approach in Eq. (5). Both PBE+D2 and PBE values evaluated perturbatively from MD@PBE and MD@PBE+D2, respectively, are close to their "exact" values, demonstrating the reliability of our approach. The $\mathrm{PBE}+\mathrm{D} 2$ perturbative estimate is slightly worse, showing the importance of starting from an approach that correctly samples the key adsorption region of phase space.

From a practical point of view, this brute force application of perturbation theory involves the reevaluation of the energy for thousands of configurations [specifically, the reevaluation of the electronic part of Eq. (2)]. If, starting from MD@PBE or $\mathrm{MD} @ \mathrm{PBE}+\mathrm{D} 2$, we applied this perturbative procedure to compute the RPA enthalpy of adsorption, the corresponding computational time would amount to several millions of CPU hours in our supercomputer center. While technically feasible, such high demands are of limited practical interest.

We now proceed to show that it is possible to reduce the number of times the electronic part of Eq. (2) is recomputed to a very small fraction of the total number of configurations. This is achieved by finding a semianalytic model of $P_{V}^{H}(E)$, and using it to build an approximate model of $P_{V^{\prime}}^{H^{\prime}}(E)$ by assuming that the distributions generated by $H$ and $H^{\prime}$ (e.g., $H_{\mathrm{PBE}+\mathrm{D} 2}$ and $H_{\mathrm{RPA}}$, as used here) are similar. We employ the following procedure to approximately reconstruct the correct high-level energy distribution $P_{H^{\prime}}\left(V^{\prime}\right)$ and corresponding average values:

Step 1: MD driven by the Hamiltonian $H(\mathbf{q}, \mathbf{p})$ is performed to generate the $P_{H}^{V}(E)$ distribution [Eq. (4)].
Step 2: $P_{H}^{V}(E)$ is approximated by a set of $N_{G}$ Gaussian functions with $3 N_{G}$ fitting parameters $\left\{h_{i}, w_{i}, \epsilon_{i}\right\}$,

$$
\tilde{P}_{H}^{V}(E)=\frac{1}{M} \sum_{i=1}^{N_{G}} h_{i} \exp \left\{-\frac{\left(E-\epsilon_{i}\right)^{2}}{2 w_{i}^{2}}\right\},
$$

with normalization constant $M=\sum_{i=1}^{N_{G}} \sqrt{2 \pi} h_{i} w_{i}$. Parameters are selected to represent key statistical features of the distribution. The tilde introduced in Eq. (7) emphasizes the approximate character of the probability distribution. It is crucial here to forbid any single or a limited number of Gaussians to dominate; for this reason the contribution of each single Gaussian is limited to a certain threshold. We note that this representation of potential energy density is related to the kernel density estimation method of probability density functions [61]. Technical details on the fitting with Gaussian functions are further discussed in the Supplemental Material [59].

Step 3: $N_{G}$ configurations with positions $\mathbf{q}_{i}$ are selected from the ensemble of step 1 to satisfy

$$
V\left(\mathbf{q}_{i}\right) \in\left[\epsilon_{i}-\Delta, \epsilon_{i}+\Delta\right],
$$

where we use $\Delta=0.02 \mathrm{kcal} / \mathrm{mol}$. Since several timeuncorrelated configurations can satisfy Eq. (8), the specific configuration $\mathbf{q}_{i}$ is randomly chosen with uniform probability. This is a reasonable procedure since configurations with the same energy $\left(\epsilon_{i}\right)$ have equal probability to be sampled. For the selected configurations $\mathbf{q}_{i}$ a different (higher) level of theory is used to evaluate the energies $V^{\prime}\left(\mathbf{q}_{i}\right)$ to obtain $\Delta V_{i} \equiv$ $\Delta V\left(\mathbf{q}_{i}\right)=V^{\prime}\left(\mathbf{q}_{i}\right)-V\left(\mathbf{q}_{i}\right)$.

Step 4: Next, we calculate

$$
\epsilon_{i}^{\prime}=\epsilon_{i}+\Delta V_{i}, \quad h_{i}^{\prime}=h_{i} \exp \left\{-\beta \Delta V_{i}\right\},
$$

and use them to obtain

$$
\tilde{P}_{H^{\prime}}^{V^{\prime}}(E)=\frac{1}{M^{\prime}} \sum_{i=1}^{N_{G}} h_{i}^{\prime} \exp \left\{-\frac{\left(E-\epsilon_{i}^{\prime}\right)^{2}}{2 w_{i}^{2}}\right\},
$$

where $M^{\prime}=\sum_{i=1}^{N_{G}} \sqrt{2 \pi} h_{i}^{\prime} w_{i}$. This equation follows from Eqs. (5) and (7), with reweighting factor $\exp \{-\beta \Delta V\}$.

Step 5: Finally, we determine the estimate of $\left\langle V^{\prime}\right\rangle_{H^{\prime}}$ by combining Eq. (10) with Eq. (4). The accuracy of this ensemble average can be evaluated by computing the standard error according to Eq. (S17) (see Supplemental Material [59]). If a higher level of accuracy is required, the original set of selected 
configurations can be extended to decrease the statistical error of the final result, as done later.

We label the five-step procedure described above by the acronym "selPT," which stands for perturbation theory on selected configurations. In order for this approach to be useful, statistical convergence should be achieved for $N_{G} \ll N_{\mathrm{MD}}$, where $N_{\mathrm{MD}}$ denotes the total number of MD steps. This is not an unreasonable expectation, as the configurations produced within a certain MD simulation are not completely independent due to the finite correlation time. Below, we show that even $N_{G}=60$ configurations can be sufficient for a reasonable estimate and $N_{G}=180$ can provide highly accurate results-both are vast improvements on the $N_{\mathrm{MD}} \approx 190000$ runs required to recompute a full MD trajectory. Such a dramatic reduction is possible because $\tilde{P}_{H^{\prime}}^{V^{\prime}}(E)$ is a smooth, representative function that captures the important properties of the true distribution $P_{H^{\prime}}^{V^{\prime}}(E)$.

To establish the accuracy and the efficiency of the selPT procedure we come back to the example of $\mathrm{CH}_{4}$ adsorbed on chabazite. Before computing RPA adsorption energies, the approach is validated using PBE and PBE+D2: First, the "exact" distribution $P_{\mathrm{H}_{\mathrm{PEE}+\mathrm{D} 2}}^{V_{\mathrm{PB}}}$ generated by the previously described MD@PBE+D2 simulation is fitted by 60 Gaussianshaped functions [Eq. (7)]; second, 60 configurations $\mathbf{q}_{i}$ are chosen in order to satisfy the condition in Eq. (8); finally, Eq. (10) is used to evaluate $\tilde{P}_{H_{\mathrm{PBE}}}^{V_{\mathrm{BBE}}}$ and the corresponding ensemble averages. The procedure is repeated three times to generate three uncorrelated sets containing 60 configurations each, that will be called SET1@PBE+D2, SET2@PBE+D2, and SET3@PBE+D2. This is helpful to study the behavior of the selPT method as a function of the number of configurations. Indeed, the adsorption enthalpies have been computed using the three sets with 60 configurations, from the three sets with 120 configurations obtained by merging pairs of the original sets (SET1+2@PBE+D2, SET2+3@PBE+D2, and SET1+3@PBE+D2), and with a set that includes all the configurations $(\mathrm{SET} 1+2+3 @ \mathrm{PBE}+\mathrm{D} 2)$. Since the enthalpy of adsorption Eq. (6) requires ensemble averages for the three different systems $(S, A, S+A)$, the selPT procedure is applied independently on each one of them.

Figure 1 demonstrates the fitting quality. The middle panel demonstrates the ability of selPT to reproduce key features of the sampling data, and to perturb them using a more accurate method, by comparing $P_{H}^{V}$ from the full MD@PBE+D2 run, with its parametrized distribution $\tilde{P}_{H}^{V}$ and RPA counterpart $\tilde{P}_{H^{\prime}}^{V^{\prime}}$ approximated from Eq. (10) using SET2@PBE+D2. The right panel shows a phase-space diagram for the full MD run, and the 60 samples selected by selPT, reported in terms of variables representing the speed of $\mathrm{CH}_{4}$ and its distance from the surface of the chabazite. Note that selPT samples the probability distribution accurately, by design; as shown in the right panel of Fig. 1, a representative part of phase space is also covered for the system under consideration.

The row corresponding to PBE in Table I shows the practical reliability of selPT in computing enthalpies of adsorption $\Delta_{\text {ads }} H(T)$. Specifically, the similarity of the energies across all calculations highlights the success of the approach. If our trajectories sampled an overly limited set of configurations (whether through a too-short trajectory or too-inaccurate functional), or if our selections sets were too poor, the numbers would differ significantly across columns. The consistency of the values justifies the length and reasonableness of our initial AIMD calculations, and the accuracy of the selection process.

The bottom row of Table I shows RPA enthalpies of adsorption, which would be basically impossible to obtain using a full AIMD treatment due to the inherent cost of RPA (full results considering all the possible combinations of sets are provided in Tables S9 and S10 of the Supplemental Material [59]). Numerical results are based on the RPA implementation in the VASP code [28] and computational parameters are provided in the Supplemental Material [59]. The statistically most accurate selPT estimate of the RPA enthalpy of adsorption is obtained from the largest set SET1+2+3@PBE+D2 $\left(N_{G}=180\right)$ and provides a value of $-4.38 \pm 0.39 \mathrm{kcal} / \mathrm{mol}$, in excellent agreement with the experimental value of -4.06 $\mathrm{kcal} / \mathrm{mol}[62,63]$. From Table I (and Tables S9 and S10) it can be noticed that the errors present a clear trend to decrease as a function of the number of configurations included in the selPT set. In Table S9 it is shown that RPA results from as few as 60 samples are all within $0.92 \mathrm{kcal} / \mathrm{mol}$ of one another, and within $0.83 \mathrm{kcal} / \mathrm{mol}$ of experiment. This is already a considerable improvement with respect to the PBE and PBE+D2 estimates, which present deviations of at least $2 \mathrm{kcal} / \mathrm{mol}$ well beyond the chemical accuracy.

Key for this high level of accuracy is that the selPT calculations are based on a reasonable $\mathrm{PBE}+\mathrm{D} 2$ trajectory which, unlike PBE, samples phase space similarly to RPA. This ensures that the perturbation from $\mathrm{PBE}+\mathrm{D} 2$ to RPA is correctly biased to the weak $\mathrm{vdW}$ interaction between the zeolite and molecule. This intuitive point is further discussed in a quantitative way in the Supplemental Material [59] where RPA results from a PBE starting point are presented (Secs. SIII and SIV) and it is shown how PBE leads to a significantly different sampling of the configurational space with respect to PBE+D2 and RPA (Sec. SV).

To further establish the accuracy and reliability of the selPT approach, a second system is discussed, $\mathrm{CO}_{2}$ adsorbed in protonated chabazite. Computational details are analogous to those of $\mathrm{CH}_{4}$ in chabazite. The adsorption enthalpy of $\mathrm{CO}_{2}$ is sizably larger than that of $\mathrm{CH}_{4}$, with an experimental value of $-8.41 \mathrm{kcal} / \mathrm{mol}$ at room temperature [64]. While the PBE+D2 result deviates from this value by 1.3 $\mathrm{kcal} / \mathrm{mol}$, our most accurate estimate from the largest set SET1+2+3@PBE+D2 provides an highly accurate enthalpy of adsorption of $-8.74 \pm 0.39 \mathrm{kcal} / \mathrm{mol}$. Detailed results for all the test sets are presented in Table $\mathrm{S} 9\left(N_{G}=60\right)$ and $\mathrm{S} 10$ $\left(N_{G}=120\right)$ in the Supplemental Material [59]. The errors are analogous to those for adsorbed $\mathrm{CH}_{4}$; by considering that the adsorption enthalpy of $\mathrm{CO}_{2}$ is about double that of $\mathrm{CH}_{4}$, the relative error in this case decreases much more rapidly as a function of the set size and $N_{G}=60$ provides already an accuracy to within $\pm 10 \%$.

From a practical point of view, the selPT approach has been instrumental to achieve the computation of RPA enthalpies of adsorption. By decreasing the number of configurations to 60 , the RPA results are obtained in a CPU time that is 
only about three to four times the CPU time involved in the full MD@PBE calculation, or around ten times for the more accurate simulation with 180 configurations.

In conclusion, we introduced the selPT method to compute ensemble averages of energies and, more generally, energy distributions for correlated wave-function methods. As proof of principle, this methodology was applied to compute the finite-temperature adsorption energy of $\mathrm{CH}_{4}$ and $\mathrm{CO}_{2}$ in protonated chabazite at the RPA level of theory, where it successfully reproduced known experimental values within chemical accuracy. selPT is not limited to the RPA but could be interfaced with other sophisticated approximations (e.g., MP2, double hybrids, or coupled-cluster theory), as long as computations on few tens of configurations can be afforded.

Beyond providing an accurate and efficient methodology to address challenging problems in physics and material science, selPT could be also used to develop finite-temperature test sets from high-level theories, to test and develop new DFT functionals and semiclassical models.

This work was supported by Agence Nationale de la Recherche under Grant No. ANR-15-CE29-0003-01. T.B. acknowledges support from Slovak Research and Development Agency under the Contracts No. APVV-15-0105 and No. VEGA-1/0777/19. D.R., T.G., and S.L. acknowledge support from the French PIA project Lorraine Université d'Excellence. The results of this research have been achieved using GENCI-CCRT/CINES computational resources under Grants No. A0030805106 and No. A0040910433, the DECI resource ARCHER based in the United Kingdom with support from the PRACE aisbl, and the Computing Center of the Slovak Academy of Sciences acquired in Projects ITMS No. 26230120002 and No. 26210120002 supported by the Research and Development Operational Program funded by the ERDF.
[1] R. Car and M. Parrinello, Phys. Rev. Lett. 55, 2471 (1985).

[2] W. Kohn and L. J. Sham, Phys. Rev. 140, A1133 (1965).

[3] A. J. Cohen, P. Mori-Sánchez, and W. Yang, Chem. Rev. 112, 289 (2011).

[4] C. Møller and M. S. Plesset, Phys. Rev. 46, 618 (1934).

[5] R. J. Bartlett and M. Musial, Rev. Mod. Phys. 79, 291 (2007).

[6] A. Szabo and N. S. Ostlund, Modern Quantum Chemistry: Introduction to Advanced Electronic Structure Theory (Courier Corporation, North Chelmsford, MA, 2012).

[7] M. Marsman, A. Grüneis, J. Paier, and G. Kresse, J. Chem. Phys. 130, 184103 (2009).

[8] A. Dixit, J. Claudot, S. Lebègue, and D. Rocca, J. Chem. Phys. 146, 211102 (2017).

[9] C. Pisani, L. Maschio, S. Casassa, M. Halo, M. Schütz, and D. Usvyat, J. Comput. Chem. 29, 2113 (2008).

[10] M. Del Ben, J. Hutter, and J. VandeVondele, J. Chem. Theory Comput. 8, 4177 (2012).

[11] G. H. Booth, A. Grüneis, G. Kresse, and A. Alavi, Nature (London) 493, 365 (2013).

[12] J. McClain, Q. Sun, G. K.-L. Chan, and T. C. Berkelbach, J. Chem. Theory Comput. 13, 1209 (2017).

[13] Q. Sun, T. C. Berkelbach, N. S. Blunt, G. H. Booth, S. Guo, Z. Li, J. Liu, J. D. McClain, E. R. Sayfutyarova, S. Sharma et al., WIREs Comput. Mol. Sci. 8, e1340 (2018).

[14] D. Bohm and D. Pines, Phys. Rev. 92, 609 (1953).

[15] M. Gell-Mann and K. A. Brueckner, Phys. Rev. 106, 364 (1957).

[16] D. C. Langreth and J. P. Perdew, Solid State Commun. 17, 1425 (1975).

[17] J. F. Dobson and J. Wang, Phys. Rev. Lett. 82, 2123 (1999).

[18] F. Furche, Phys. Rev. B 64, 195120 (2001).

[19] H. Eshuis and F. Furche, J. Phys. Chem. Lett. 2, 983 (2011).

[20] A. Heßelmann and A. Görling, Mol. Phys. 109, 2473 (2011).

[21] X. Ren, P. Rinke, C. Joas, and M. Scheffler, J. Mater. Sci. 47, 7447 (2012).

[22] D. C. Langreth and J. P. Perdew, Phys. Rev. B 15, 2884 (1977).

[23] G. E. Scuseria, T. M. Henderson, and D. C. Sorensen, J. Chem. Phys. 129, 231101 (2008).
[24] B. Mussard, D. Rocca, G. Jansen, and J. G. Angyán, J. Chem. Theory Comput. 12, 2191 (2016).

[25] M. Del Ben, J. Hutter, and J. VandeVondele, J. Chem. Phys. 143, 054506 (2015).

[26] A. Marini, P. Garcia-Gonzalez, and A. Rubio, Phys. Rev. Lett. 96, 136404 (2006).

[27] S. Lebègue, J. Harl, T. Gould, J. G. Ángyán, G. Kresse, and J. F. Dobson, Phys. Rev. Lett. 105, 196401 (2010).

[28] J. Harl and G. Kresse, Phys. Rev. B 77, 045136 (2008).

[29] D. Lu, Y. Li, D. Rocca, and G. Galli, Phys. Rev. Lett. 102, 206411 (2009).

[30] J. Harl, L. Schimka, and G. Kresse, Phys. Rev. B 81, 115126 (2010).

[31] F. Kaoui and D. Rocca, J. Phys.: Condens. Matter 28, 035201 (2016).

[32] X. Ren, P. Rinke, and M. Scheffler, Phys. Rev. B 80, 045402 (2009).

[33] L. Schimka, J. Harl, A. Stroppa, A. Grüneis, M. Marsman, F. Mittendorfer, and G. Kresse, Nat. Mater. 9, 741 (2010).

[34] F. Göltl, A. Grüneis, T. Bučko, and J. Hafner, J. Chem. Phys. 137, 114111 (2012).

[35] F. Furche and T. van Voorhis, J. Chem. Phys. 122, 164106 (2005).

[36] A. Heßelmann and A. Görling, Phys. Rev. Lett. 106, 093001 (2011).

[37] J. E. Bates and F. Furche, J. Chem. Phys. 139, 171103 (2013).

[38] T. Olsen and K. S. Thygesen, Phys. Rev. B 86, 081103(R) (2012).

[39] X. Ren, P. Rinke, G. E. Scuseria, and M. Scheffler, Phys. Rev. B 88, 035120 (2013).

[40] D. Lu, J. Chem. Phys. 140, 18A520 (2014).

[41] N. Colonna, M. Hellgren, and S. de Gironcoli, Phys. Rev. B 90 , 125150 (2014).

[42] A. Dixit, J. Claudot, S. Lebègue, and D. Rocca, J. Chem. Theory Comput. 13, 5432 (2017).

[43] M. Hellgren, N. Colonna, and S. de Gironcoli, Phys. Rev. B 98, 045117 (2018).

[44] A. Dixit, J. Claudot, T. Gould, S. Lebègue, and D. Rocca, Phys. Rev. B 97, 115104 (2018). 
[45] K. Liao and A. Grüneis, J. Chem. Phys. 145, 141102 (2016).

[46] V. V. Speybroeck, K. Hemelsoet, L. Joos, M. Waroquier, R. G. Bell, and C. R. A. Catlow, Chem. Soc. Rev. 44, 7044 (2015).

[47] S. Chibani, M. Chebbi, S. Lebègue, T. Bučko, and M. Badawi, J. Chem. Phys. 144, 244705 (2016).

[48] T. Bučko, S. Chibani, J.-F. Paul, L. Cantrel, and M. Badawi, Phys. Chem. Chem. Phys. 19, 27530 (2017).

[49] S. Chibani, M. Badawi, T. Loiseau, C. Volkringer, L. Cantrel, and J.-F. Paul, Phys. Chem. Chem. Phys. 20, 16770 (2018).

[50] L. Grajciar, C. J. Heard, A. A. Bondarenko, M. V. Polynski, J. Meeprasert, E. A. Pidko, and P. Nachtigall, Chem. Soc. Rev. 47, 8307 (2018).

[51] H. Babaei and C. E. Wilmer, Phys. Rev. Lett. 116, 025902 (2016).

[52] K. Adil, Y. Belmabkhout, R. S. Pillai, A. Cadiau, P. M. Bhatt, A. H. Assen, G. Maurin, and M. Eddaoudi, Chem. Soc. Rev. 46, 3402 (2017).

[53] D. S. Sholl and R. P. Lively, Nat. News 532, 435 (2016).

[54] M. Del Ben, J. Hutter, and J. VandeVondele, J. Chem. Phys. 143, 102803 (2015).
[55] B. Ramberger, T. Schäfer, and G. Kresse, Phys. Rev. Lett. 118, 106403 (2017).

[56] M. Bokdam, J. Lahnsteiner, B. Ramberger, T. Schäfer, and G. Kresse, Phys. Rev. Lett. 119, 145501 (2017).

[57] C. Chipot and A. Pohorille, Free Energy Calculations: Theory and Applications in Chemistry and Biology (Springer, Berlin, 2016).

[58] S. Grimme, J. Comput. Chem. 27, 1787 (2006).

[59] See Supplemental Material at http://link.aps.org/supplemental/ 10.1103/PhysRevMaterials.3.040801 for computational details, fitting procedure description, error estimation, and analysis of impact of sampling method on selPT results.

[60] G. Kresse and J. Furthmüller, Phys. Rev. B 54, 11169 (1996); G. Kresse and D. Joubert, ibid. 59, 1758 (1999).

[61] E. Parzen, Ann. Math. Stat. 33, 1065 (1962).

[62] G. Piccini, M. Alessio, J. Sauer, Y. Zhi, Y. Liu, R. Kolvenbach, A. Jentys, and J. A. Lercher, J. Phys. Chem. C 119, 6128 (2015).

[63] Y. Luo, H. H. Funke, J. L. Falconer, and R. D. Noble, Ind. Eng. Chem. Res. 55, 9749 (2016).

[64] T. D. Pham, Q. Liu, and R. F. Lobo, Langmuir 29, 832 (2013). 